\title{
Esclerose Múltipla: duas apresentações clínicas, uma só doença!
}

\author{
Multiple Sclerosis: two clinical presentations, a single disease!
}

\section{Esclerosis múltiple: dos presentaciones clínicas, una sola enfermedad!}

Ana Margarida Ferreira da Silva. Unidade de Saúde Familiar das Ondas, Aces Porto IV. Póvoa de Varzim, Vila do Conde, Portugal. amargferreiradasilva@gmail.com (Autora correspondente)

Francisco Pinto da Costa. Unidade de Saúde Familiar das Ondas, Aces Porto IV. Póvoa de Varzim, Vila do Conde, Portugal. franciscopintodacost@ gmail.com

Miguel Baptista. Hospital Pedro Hispano. Unidade Local de Saúde Matosinhos. Portugal. mikefyssas@gmail.com

Teresa Febra. Unidade de Saúde Familiar das Ondas, Aces Porto IV. Póvoa de Varzim, Vila do Conde, Portugal. teresafebraondas@gmail.com Carla Morna. Unidade de Saúde Familiar das Ondas, Aces Porto IV. Póvoa de Varzim, Vila do Conde, Portugal. carlamornaondas@gmail.com

\section{Resumo}

Objetivo: 0 presente relato de caso pretende demonstrar a diversidade de apresentações clínicas, a evolução dos sintomas e o papel do médico da atenção primária no diagnóstico e seguimento de pacientes com esclerose múltipla e seus familiares. Descrição dos casos: duas mulheres, de $31 \mathrm{e}$ 28 anos, caucasianas, inseridas em famílias nucleares (fases IV e II do ciclo de Duvall, respectivamente) pertencentes à classe média de Graffar. A primeira inicia um quadro insidioso de parestesias das mãos com melhoria em 2 meses. Passado um ano, apresenta-se com dificuldade em elevar as pálpebras e desequilíbrio marcado. A segunda apresenta diminuição súbita da acuidade visual à direita, tendo sido diagnosticada uma neurite óptica. Ambas foram diagnosticadas com esclerose múltipla. Conclusão: a esclerose múltipla é uma doença inflamatória crônica, desmielinizante e degenerativa do sistema nervoso central, que se revela de forma heterogênea. Torna-se importante ao médico de família saber como lidar com as incertezas diagnósticas.

\section{Abstract}

Objective: this case report aims to demonstrate the diversity of clinical presentations, the symptoms evolution and the role of the primary care physician in the diagnosis and management of patients with multiple sclerosis, and their families. Case descriptions: two women, 31 and 28 years old, Caucasian, inserted within nuclear families (phases II and IV of the Duvall' cycle, respectively) belonging to the middle class of Graffar. The first one starts an insidious symptom of paraesthesia of the hands with improvement in 2 months. Within a year, she presents with difficulty raising the eyelids and marked imbalance. The second one presents sudden loss of visual acuity on the right, having been diagnosed with optic neuritis. Both were diagnosed with multiple sclerosis. Conclusion: multiple sclerosis is a chronic inflammatory, degenerative and demyelinating disease of the central nervous system that manifests heterogeneously. It is important for the family doctor to know how to deal with diagnostic uncertainties.

\section{Resumen}

Objetivo: el presente reporte de caso tiene como objetivo demostrar la diversidad de presentaciones clínicas, la evolución de los síntomas y el rol del médico de atención primaria en el diagnóstico y manejo de pacientes con esclerosis múltiple y sus familiares. Descripción de los casos: dos mujeres, 31 y 28 años de edad, de raza caucásica, insertados en las familias nucleares (fases II y IV del Ciclo de Duvall, respectivamente) que pertenecen a la clase media Graffar. La primera inicia con síntomas insidiosos de parestesias de las manos, con una mejoría en 2 meses. Dentro de un año, se presenta con dificultad para levantar los párpados y marcado desequilibrio. La segunda presenta pérdida súbita de la agudeza visual a la derecha y fue diagnosticado con neuritis óptica. Ambas fueron diagnosticadas con esclerosis múltiple. Conclusión: la esclerosis múltiple es una enfermedad inflamatoria crónica, degenerativa del sistema nervioso central y desmielinizante, que se revela heterogéneamente. Es importante para el médico familiar conocer las incertidumbres diagnósticas.
Palavras-chave:

Esclerose Múltipla Sistema Nervoso Central Parestesia Neurite Óptica Atenção Primária à Saúde

\section{Keywords:}

Multiple Sclerosis Central Nervous System Paresthesia Optic Neuritis Primary Health Care

\section{Palabras clave:} Esclerosis Múltiple Sistema Nervioso Central Parestesia Neuritis Óptica Atención Primaria de Salud 


\section{Introdução}

A esclerose múltipla (EM) surge frequentemente em jovens adultos, entre os 20 e os 40 anos, com o dobro da incidência no sexo feminino e em pessoas do norte da Europa. ${ }^{1}$ Dados da Organização Mundial de Saúde (OMS) estimam que, em todo o mundo, existam cerca de 2,1 milhóes de pessoas com EM e, em Portugal, mais de 5000 pessoas. ${ }^{2}$ A EM é uma doença inflamatória crônica, desmielinizante e degenerativa do sistema nervoso central que interfere no controle de funçóes como a visão, a locomoçáo e o equilíbrio. ${ }^{1}$ Trata-se de uma doença heterogênea, com várias características clínicas e patológicas, refletindo diferentes caminhos de lesão tecidual, permanecendo ainda desconhecida a sua causa. O início pode ser abrupto ou insidioso, sendo que alguns pacientes apresentam sintomas tão comuns que não despertam a atenção médica durante meses ou anos.

A inexistência de sinais e sintomas patognomônicos de EM torna difícil o seu diagnóstico. Geralmente, este se baseia nas apresentações clínicas mais características, que incluem diplopia, parestesias, fraqueza, incontinência ou retenção urinária, depressão, tremor de ação e hiperreflexia. ${ }^{1}$ A demonstração de lesóes disseminadas no espaço e no tempo, por meio de ressonância magnética nuclear (RMN), complementa os critérios de diagnóstico. ${ }^{3} \mathrm{~A}$ RMN demonstrou ser altamente sensível na detecção de placas clinicamente silenciosas $s^{4,5} \mathrm{e}$, por conseguinte, os resultados da presente modalidade de imagem estáo incluídos nos critérios de diagnóstico de McDonald. ${ }^{3,6,7}$ Os testes sorológicos e a punção lombar podem ser usados para exclusão de outras patologias. ${ }^{3,4}$

O objetivo da apresentação destes dois casos clínicos é alertar para a variabilidade de apresentaçóes clínicas de uma patologia pouco frequente, que pode surgir em um curto espaço de tempo ao médico da Atenção Primária, necessitando de um olhar mais atento e global perante o presente e o futuro do paciente e de sua família. Obteve-se consentimento informado junto aos pacientes e também a aprovação junto à coordenadoria local da USF das Ondas, parte da Administração Regional de Saúde do Norte (ARS Norte) em 14/05/2012.

\section{Descrição dos casos}

\section{Caso 1}

A.C.F., 31 anos, sexo feminino, caucasiana, talhante (açougueiro, no Brasil), com o $6^{\circ}$ ano de escolaridade. Pertencente a uma família nuclear e altamente funcional, de acordo com a aplicação do Apgar Familiar de Smilkstein, constituída por A.C.F., o marido e o filho (Figura 1). Sem antecedentes pessoais relevantes, em termos familiares o marido é portador de paramiloidose, tendo sido submetido a transplante hepático.

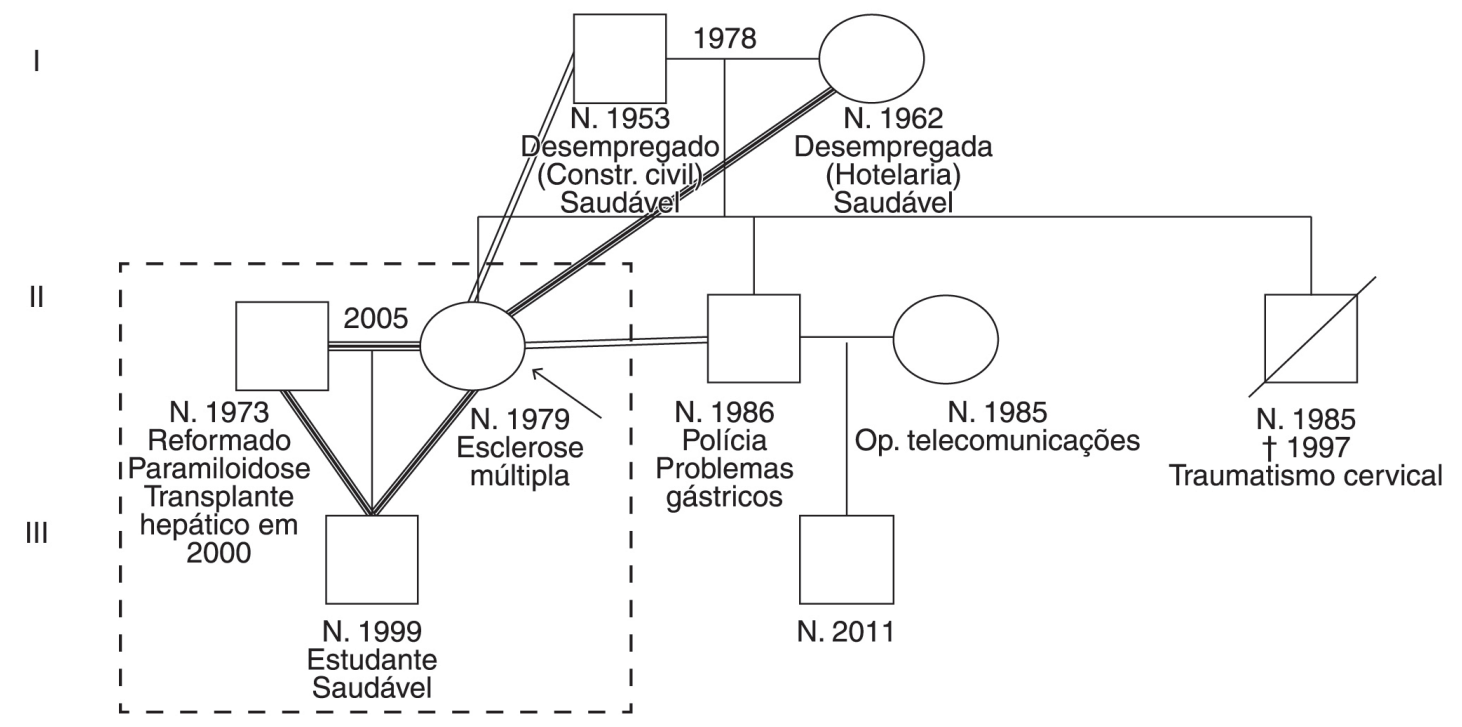

Figura 1. Genograma e Psicofigura de Mitchell da família do Caso 1 (realizado a 12 de outubro de 2011). 


\section{História da Doença Atual}

A paciente recorre à demanda espontânea da sua médica de família a 20 de abril de 2010, por quadro insidioso de parestesias nos dedos das mãos há 1 semana, sem outros sintomas associados. O exame neurológico foi normal, sem déficits motores focais, reflexos osteotendinosos normais, sem noção de assimetrias sensitivas, nem níveis sensitivos objetiváveis. Tendo em vista a ausência de antecedentes e a profissáo da paciente considerou-se a hipótese de se tratar de uma síndrome de coluna cervical associada a parestesias nos dedos das mãos. A paciente foi aconselhada a fazer correçóes posturais e medicada com anti-inflamatório, tendo sido solicitada radiografia da coluna cervical.

Cerca de 2 semanas depois, a paciente retorna à consulta com o resultado da radiografia da coluna, que se encontrava sem alteraçóes. A paciente refere que, além das parestesias nos dedos das mãos, manifesta dor nos ombros, sensação de depressão com insônia inicial e incapacidade para realização das atividades da vida diária, há uma semana. O exame neurológico manteve-se inalterado, e o exame do ombro não revelou sinais inflamatórios associados. Uma vez que as queixas se mantiveram inespecíficas e associadas a sintomas depressivos, adicionou-se um relaxante muscular e um antidepressivo.

Em 14 de maio de 2010, a paciente recorre novamente à demanda espontânea, por infecção do trato respiratório superior, para a qual foi medicada com antibiótico. Dez dias depois, a paciente regressa por aparecimento de erupção cutânea localizada, com um dia de evolução. Sem alergias medicamentosas conhecidas. Mantendo queixas de formigamento nos dedos das mãos. Ao exame físico é observado um exantema máculo-papular disperso na face (asa de borboleta), nos membros superiores e coxas, tendo sido medicada com corticoide e anti-histamínico e solicitado estudo imunológico para exclusão de lúpus eritematoso sistêmico (anticorpos antinucleares (FAN), velocidade de hemossedimentação (VHS) e anticorpos anti-DNA nativo [anti-dsDNA]), que se revelaram normais. Levando-se em conta essa evolução, a paciente foi reavaliada em 1 de junho, com melhora da erupção cutânea e ausência de formigamento. A paciente foi posteriormente acompanhada, sem intercorrências relevantes.

A 15 de março de 2011, recorre novamente à consulta por, há 2 meses, sentir dificuldade em elevar as pálpebras ou olhar para cima sustentadamente. Nega dor com o movimento ocular ou periorbitária, diplopia, olho vermelho, prurido, secreção ocular, traumatismo, cefaleias, tonturas, náuseas ou vômitos. Já havia sido avaliada por oftalmologista, tendo sido recomendada correção ortótica para miopia. Ao exame neurológico verifica-se ptose bilateral e limitação bilateral da supra e infraversão conjugada e da convergência ocular. Com base na evolução prévia, a paciente é referenciada para o serviço de urgência (SU) de neurologia.

Passados dois dias a paciente retorna à consulta com carta de alta do SU referindo estudo de imagem (tomografia computorizada [TAC]) e exames de sangue sem alteraçóes, e a impressão de se tratar de uma síndrome conversiva ou miastenia gravis, forma ocular. Apresentava agravamento da sintomatologia palpebral com dificuldade na elevação das pálpebras e no olhar sustentado para cima. Com vistas a agilizar o diagnóstico, foi solicitada eletroneuromiografia (ENM).

A paciente retorna cerca de uma semana depois, a 24 de março de 2011 com, além das queixas descritas nos dois episódios anteriores, fotofobia e desequilíbrio importante ao levantar-se. A ENM encontrava-se normal. Ao exame neurológico, a paciente apresentava ptose bilateral com limitaçáo bilateral da supra e infraversão conjugada e da convergência ocular, com nistagmo multidirecional na supraversão e noção de diplopia. Prova de Romberg e marcha instáveis. Sem déficits motores ou sensitivos aparentes. A paciente foi então encaminhada novamente ao serviço de urgência de Neurologia. Desta vez, foi internada com diagnóstico provável de "Esclerose Múltipla/Vasculite do SNC”. Os exames laboratoriais revelaram bandas oligoclonais positivas no líquido cefalorraquidiano e negativas no soro e VDRL/TPHA negativo. O estudo radiológico com RMN apresentou múltiplas áreas (> 20) de hipersinal em DP, T2 e T2 Flair dispersas pelo andar supratentorial, atingindo a substância branca dos hemisférios cerebrais de localizaçấo periventricular (algumas perpendiculares ao corpo caloso e envolvendo o esplênio à direita) e subcortical, cápsulas internas, mesencéfalo, bolbo à esquerda e cordão medular no plano de D10-D11 (Figura 2). 

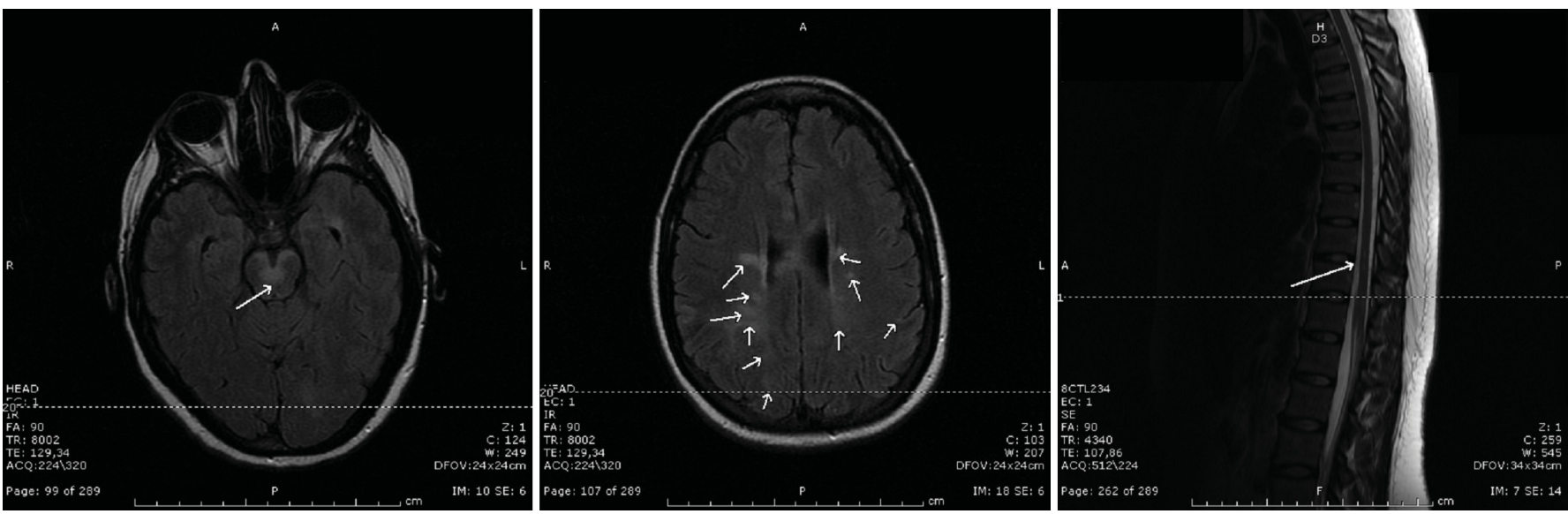

Figura 2. Imagens de RMN cerebral do Caso 1.

\section{Caso 2}

V.L.P.M.C., 28 anos, sexo feminino, caucasiana, assistente dentária, com o $12^{\circ}$ ano de escolaridade. Pertence a uma família nuclear e altamente funcional, de acordo com a aplicação do Apgar familiar de Smilkstein, constituída por V.L.P.M.C., o marido e a filha (Figura 3). Sem antecedentes pessoais relevantes. Em termos familiares, o marido tem doença de Crohn e a irmá, uma doença inflamatória intestinal inespecífica.

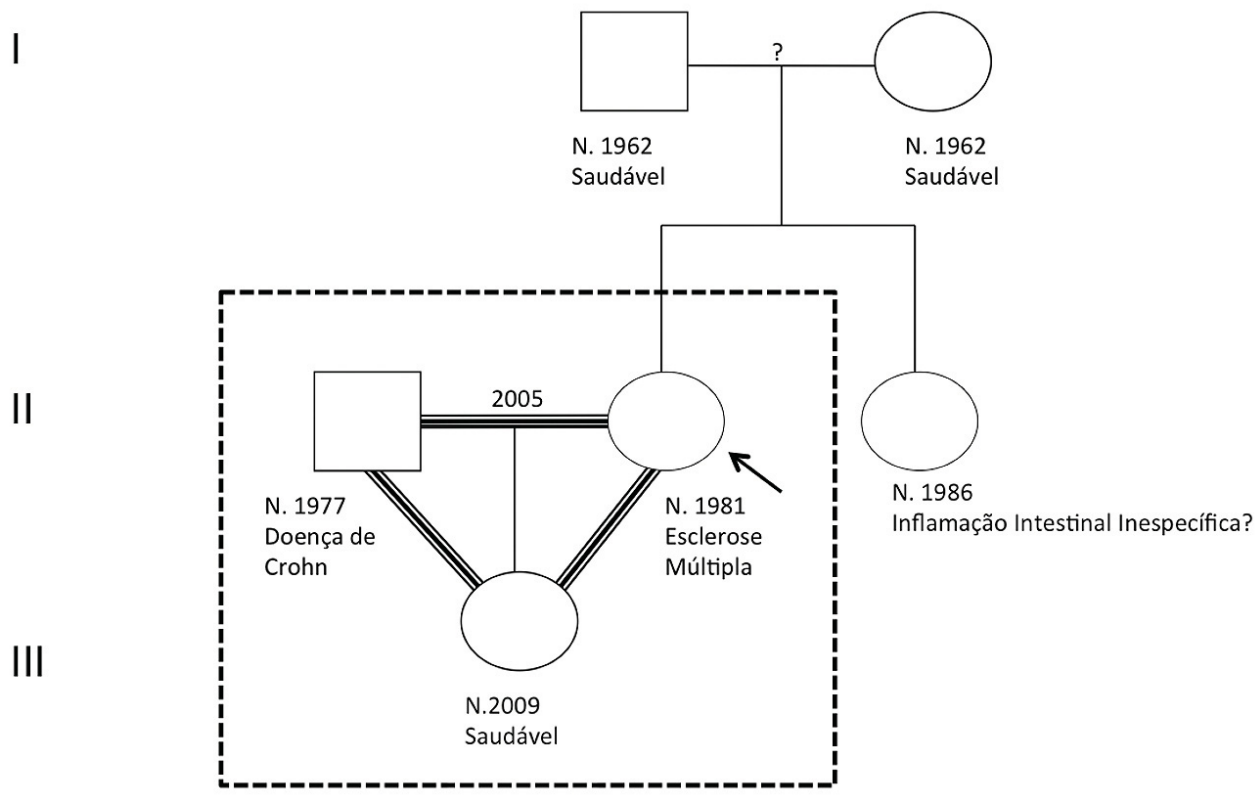

Figura 3. Genograma e Psicofigura de Mitchell da família do Caso 2 (realizado a 14 de maio de 2012).

\section{História da Doença Atual}

A paciente recorre à demanda espontânea da sua médica de família por diminuição súbita da acuidade visual do olho direito (OD) há 1 semana. Já tinha sido atendida pelo serviço de Oftalmologia, onde realizou estudo de campos visuais, que revelou defeito obliterante inferior do OD e fundoscopia que apresentou no OD "crowded disc" com bordas ligeiramente esbatidas (borradas, em português do Brasil). Ao exame objetivo do olho apresentava visão turva no campo visual inferior esquerdo do $\mathrm{OD}$. O exame neurológico não apresentava alteraçóes. 
Considerando-se a hipótese de se tratar de uma neuropatia óptica, a paciente foi referenciada ao SU de Oftalmologia. Neste foram confirmados o déficit visual e as alteraçôes à fundoscopia e, por suspeita de uma neurite óptica, a paciente foi avaliada por neurologista. Ao exame neurológico apresentava movimentos oculares preservados, ausência de dismetrias faciais, reflexos osteotendinosos vivos policinéticos nos quatro membros e cutâneo-plantares em extensão, com força muscular preservada. Por se suspeitar de uma neurite óptica como primeira manifestação de doença desmielinizante, solicitou-se uma RMN cerebral (Figura 4), onde se observaram pequenos focos de hipersinal - dois justacorticais, um peritrigonal esquerdo, dois periventriculares - e efetuou-se punção lombar que revelou bandas oligoclonais positivas no LCR e negativas no soro.
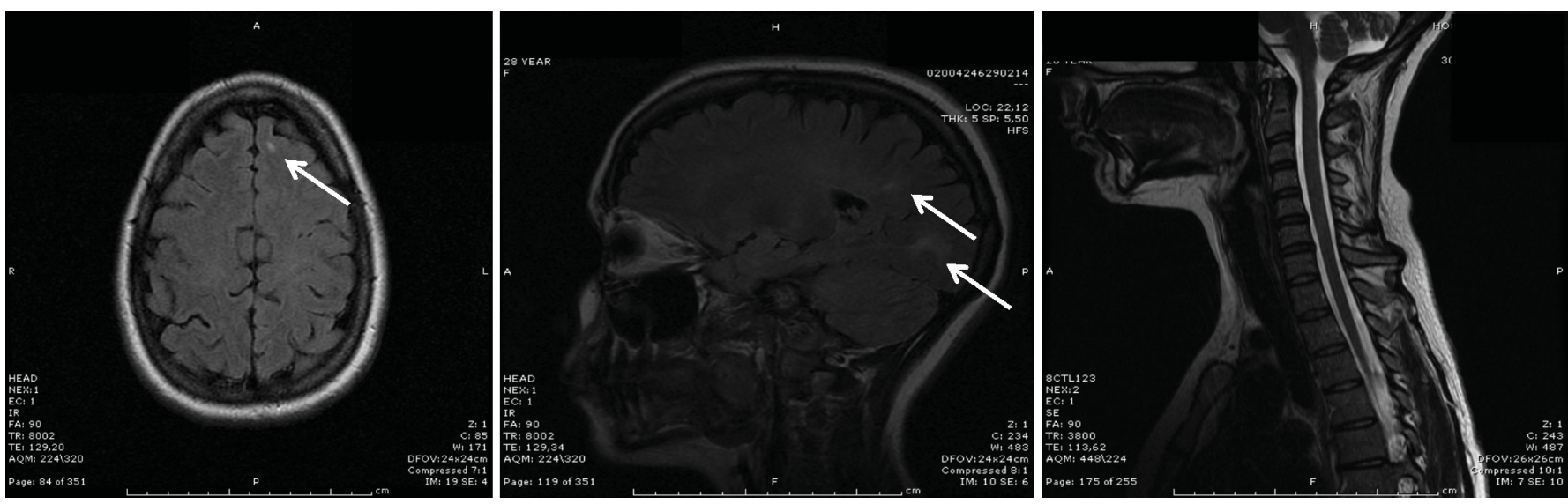

Figura 4. Imagens de RMN cerebral do Caso 2.

\section{Discussão}

A esclerose múltipla é uma doença que marca o rumo da vida de qualquer pessoa e também de sua família. Nestes casos, o médico de família deverá ser um gestor de cuidados, sendo esta uma das suas competências nucleares. Deve ainda assumir o papel de gestor de meios/recursos comunitários, de cuidados de saúde (da sua consulta e eventual referenciação) e também, se necessário, de defesa do paciente na interface de cuidados. A facilidade de acesso à consulta de demanda espontânea contribui igualmente para criar um elo de continuidade de cuidados, essencial para o raciocínio diagnóstico.

De acordo com a literatura recente, a fase inicial da doença é o melhor momento para se iniciar o tratamento, do qual deve fazer parte o Interferon beta 1-a por via intramuscular ou subcutânea, que as pacientes iniciaram após o diagnóstico.

${ }^{8-11}$ Esses casos pretendem mostrar a variabilidade de apresentaçôes clínicas da esclerose múltipla (parestesias, neurite óptica, entre outras), alertando para a inespecificidade, intermitência e diversidade de evoluçáo dos sintomas de uma patologia que coloca várias incertezas ao clínico. As incertezas diagnósticas são trabalhadas através da exclusão de "red flags" ("bandeiras vermelhas"), descartando patologias graves ou urgentes, em conjunto com os cuidados hospitalares, mais focados em fechar diagnósticos, assegurando sempre um diálogo/negociação do cuidado com o paciente/família.

Apesar de terem apresentaçôes clínicas diferentes, ambas as pacientes reúnem critérios clínicos de diagnóstico de EM na forma surto-remissão, e as imagens de RMN suportam o diagnóstico, de acordo com $\mathrm{McDonald}{ }^{3}$ A progressão da incapacidade é altamente variável mas, na maioria dos pacientes, é lenta. Assim sendo, o acompanhamento multidisciplinar, sustentado na figura do médico de família, torna-se fundamental náo somente na evidência dos sinais para o diagnóstico precoce, como para o seguimento do paciente e de sua família, dada a repercussão familiar da doença.

Nos últimos 3 anos, as pacientes têm sido avaliadas em consulta, encontrando-se assintomáticas. Apesar de o período de acompanhamento após o diagnóstico ainda ser curto, o que constitui uma limitação ao estudo, a vigilância periódica dessas pacientes permite ao médico de família explorar as expectativas pessoais e familiares e a própria evolução da doença. Após a aplicação de ferramentas como genograma, psicofigura de Mitchell e círculo de Thrower, concluímos que ambas integram famílias altamente funcionais, com grande suporte familiar. No entanto, o fato de seus cônjuges sofrerem de patologias crônicas (paramiloidose e doença de Crohn, respectivamente), além de terem filhos menores, constitui motivo de preocupação em relação ao futuro, e isso tem sido levado em consideração. 


\section{Conclusão}

O médico da atenção primária encontra-se em uma posição privilegiada para realizar uma abordagem holística aos seus pacientes, muito importante frente a quadros indiferenciados, que colocam diversas incertezas, e a esclerose múltipla é apenas um exemplo dentre muitos quadros atípicos que se apresentam a esse profissional. A prestação de cuidados longitudinais permite-lhe criar com seus pacientes uma relação que facilita o conhecimento do seu contexto biopsicossocial, e assim, uma mais adequada planificação e gestão dos seus problemas.

\section{Referências}

1. Calabresi PA. Diagnosis and management of multiple sclerosis. Am Fam Physician. 2004 Nov 15;70(10):1935-44.

2. World Health Organization. Multiple Sclerosis International Federation. Atlas: Multiple sclerosis resources in the world 2008. Geneva, Switzerland: World Health Organization; 2008.

3. Polman $\mathrm{CH}$, Reingold SC, Banwell B, Clanet M, Cohen JA, Filippi M, et al. Diagnostic criteria for multiple sclerosis: 2010 revisions to the McDonald criteria. Ann Neurol. 2011 Feb;69(2):292-302. Disponível em: http://dx.doi.org/10.1002/ana.22366

4. Paty DW, Oger JJ, Kastrukoff LF, Hashimoto SA, Hooge JP, Eisen AA, et al. MRI in the diagnosis of MS: a prospective study with comparison of clinical evaluation, evoked potentials, oligoclonal banding and CT. Neurology. 1988;38:180-5. Disponível em: http://dx.doi.org/10.1212/WNL.38.2.180

5. Tintoré M, Rovira A, Martínez MJ, Rio J, Díaz-Villoslada P, Brieva L, et al. Isolated demyelinating syndromes: comparison of different MR imaging criteria to predict conversion to clinically definite multiple sclerosis. Am J Neuroradiol 2000;21:702-6.

6. McDonald WI, Compston A, Edan G, Goodkin D, Hartung HP, Lublin FD, et al. Recommended diagnostic criteria for multiple sclerosis: guidelines from the International Panel on the diagnosis of multiple sclerosis. Ann Neurol. 2001;50:121-7. Disponível em: http://dx.doi.org/10.1002/ana.1032

7. Polman CH, Reingold SC, Edan G, Filippi M, Hartung HP, Kappos L, et al. Diagnostic criteria for multiple sclerosis: 2005 revisions to the "McDonald Criteria". Ann Neurol. 2005;58:840-6. Disponível em: http://dx.doi.org/10.1002/ana.20703

8. Coyle PK, Hartung HP. Use of interferon beta in multiple sclerosis: rationale for early treatment and evidence for dose- and frequency-dependent effects on clinical response. Mult Scler. 2002;8(Suppl 1):2-9. Disponível em: http://dx.doi.org/10.1177/135245850200800102.

9. Trapp BD, Peterson J, Ransohoff RM, Rudick R, Mork S, Bo L. Axonal transection in the lesions of multiple sclerosis. N Engl J Med. 1998;338:278-85. Disponível em: http://dx.doi.org/10.1056/NEJM199801293380502.

10. Jacobs LD, Beck RW, Simon JH, Kinkel RP, Brownsheidle CM, Murray TJ, et al. Intramuscular interferon beta-1a therapy initiated during a first demyelinating event in multiple sclerosis. CHAMPS Study Group. N Engl J Med. 2000 Sep;343:898-904. Disponível em: http://dx.doi.org/10.1056/NEJM200009283431301.

11. Comi G, Filippi M, Barkhof F, Durelli L, Edan G, Fernandex O et al. Effect of early interferon treatment on conversion to definite multiple sclerosis: a randomised study. Lancet 2001; 357: 1576-82. Disponível em: http://dx.doi.org/10.1016/S0140-6736(00)04725-5. 